\title{
Heat Diffusion in Polycrystalline Materials - A Microstructure based Material Model
}

\author{
by Sreedhar Unnikrishnakurup*, C. V. Krishnamurthy* and Krishnan Balasubramaniam* \\ * Centre for Non-Destructive Evaluation, Indian Institute of Technology Madras, sreedhar.aie@gmail.com
}

\begin{abstract}
A two-dimensional Voronoi tessellation based material model has been introduced to study the heat diffusion phenomena in polycrystalline materials consisting of a distribution of grain sizes and shapes. A method to control Voronoi cell-size distributions is presented. As the main focus of the numerical investigation is to study the role of anisotropy of the thermal conductivities in each grain of the polycrystalline material, the thermal conductivity tensor of each cell is chosen to correspond to a random crystallographic orientation. A finite element analysis of heat transport from a centrally spot-heated sample is carried out on the Voronoi tessellated domain for a fixed cell size distribution and a set of randomly oriented cells. The simulations are carried out for various sets of cell orientations. Time-Temperature profiles are generated at two orthogonally placed probes for each trial. Three inter-cell boundary conditions are examined to account for the heat transport across grains. The observed trends of the time-temperature profiles in the context of effective thermal conductivity of a polycrystalline material are discussed.
\end{abstract}

\section{Introduction}

The heterogeneous nature of materials at a certain scale has a significant impact on the macroscopic behaviour of multi-phase materials. The physics and mechanics of the underlying microstructure are responsible for the various phenomena occurring in the macroscopic level. The overall behaviour of micro-heterogeneous materials depends strongly on the size, shape, spatial distribution and properties of the microstructural constituents and their respective interfaces. The microstructural morphology and properties may also evolve under a macroscopic thermo-mechanical loading. Consequently, these microstructural influences are important for the production routes and the life performance of the material and products made thereof [1].

Depending on the amount of information available on the microstructure, the estimation of effective properties of material can be identified at different levels and is commonly known as material homogenization. The physical and mechanical properties often differ with orientation in a single crystal. In crystalline structure of atoms, the possibility for an atom to slip over one another or distort in relation to one another is easier in some directions than others. The isotropy of a material is characterized by the same material properties in all directions. Alternatively, when the properties of the material vary with different crystallographic orientations, the material is said to be anisotropic. The grain orientations are random for many polycrystalline materials before doing any work (deformation) of the material. Therefore, even if the individual grains are anisotropic, the property differences tend to average out and, overall, the material is isotropic. But when a material is formed or rolled, the grains are usually distorted and elongated in one or more directions which makes the material anisotropic.

In the last few decades, researchers showed interest in the homogenized thermal properties of microstructures [2], [3], [4]. The influence of microstructural details on the heat transport process is still not well understood. So in this paper a microstructure based material model to predict the heat diffusion in polycrystalline material is addressed.

\section{Simulation}

Microstructure generation is the first step in developing a comprehensive modeling tool for simulating microstructures. The synthetic microstructure is used to represent the microstructure of materials.

\subsection{Generation of Synthetic Microstructure}

Voronoi tessellation (VT) was used to construct the geometrical model of the microstructure. Voronoi tessellation have been traditionally used to generate a random structure which is representative of polycrystalline materials in metallurgy [6]. A lot of research have been carried out to in the past to produce numerical microstructure using Voronoi tessellation [7], [8], [9], [10], . The Voronoi tessellation divides a region into convex polygons, or cells, that fill space without overlap. The ability to model the effects of a minimum exclusion distance between the points upon which its cells are constructed is considered as the primary feature of the Voronoi tessellation [11], [12]. The main steps in the generation of microstructure are the specifying the solution boundary, generation of required number of random seed points and distributed uniformly throughout the domain followed by the Voronoi tessellation to obtain the geometry. 
Fig 1 and Fig 2 shows the Poisson Voroni Tessellation (PVT) of 100 cells generated randomly in two different trials. This uncontrolled way of generation indicates that the results are sensitive to the cell size distribution. The grain size distribution features for 2D Voronoi tessellations are fitted to three different probability distribution functions such as normal, gamma and lognormal distributions and shown in Fig 3. Gamma distribution functions and lognormal functions have been widely used to fit grain size distributions of Voronoi tessellations [11], [12].

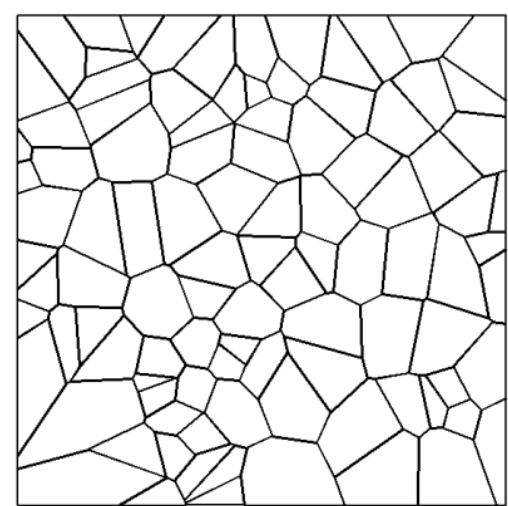

Fig 1 PVT Trial -1

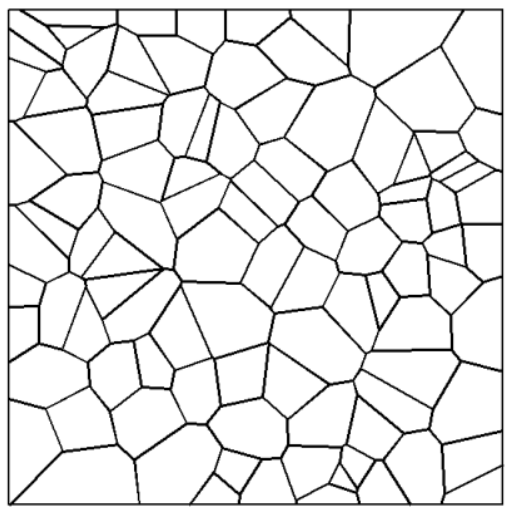

Fig 2 PVT Trial -2

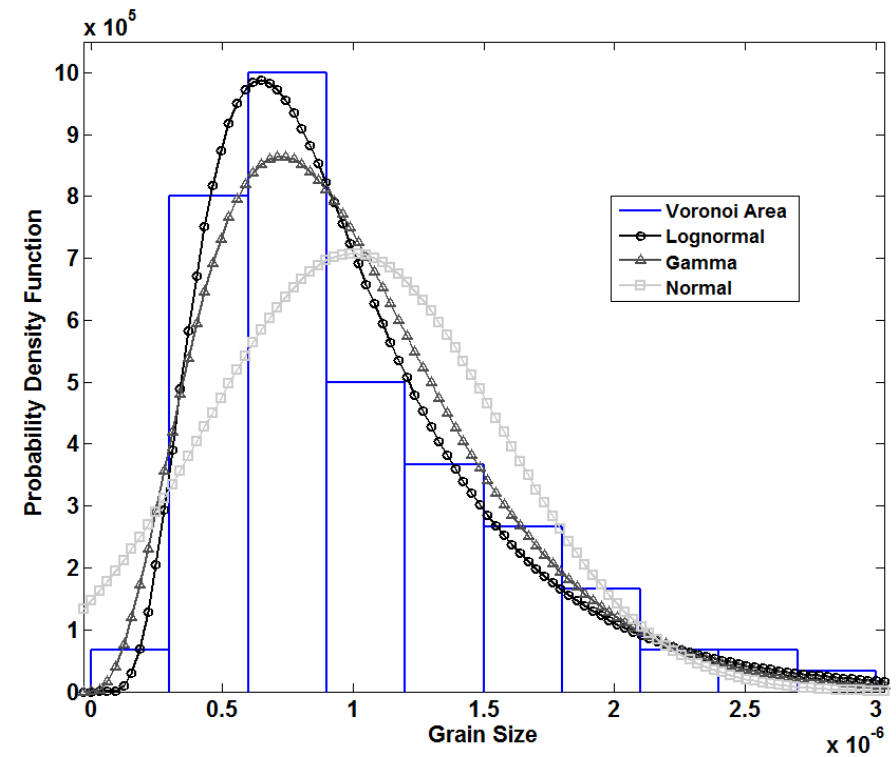

Fig 3 The histogram plot shows the grain size distribution for a Poisson Voronoi Tessellation and the three different density distribution fit

\subsubsection{Controlled Voronoi Tessellation}

In the controlled Voronoi tessellation the method is adopted from literature [12], $\mathrm{n}$ nuclei are generated in a square of are $A_{0}$. The nucleation points being created in the square by generating $x$ and $y$ coordinates independently from pseudorandom numbers. After the first point is specified, each subsequent random point is accepted only if it is greater than a minimum allowable distance $\delta$ from any existing point which ensure that the new point does not fall within an already deposited disc with diameter $\delta$ until $n$ nuclei are seeded in the square. If the size of the disc is zero, the nuclei will follow the Poisson distribution, that is distribution is completely random in space.

A non-local parameter, $\alpha$, was proposed to quantify the regularity of 2D Voronoi tessellation and is given by:

$$
\alpha=\frac{\delta}{d_{0}}
$$

Where $d_{0}$ is the distance between two adjacent seeds in an equivalent regular tessellation, i.e., a regular hexagonal 
tessellation for a 2D VT. For regular honeycomb, $\delta=d_{0}$ and $\alpha=1$ while, for a Poisson Voronoi tessellation $\delta=0$ and $\alpha=0$. For $\delta<d_{0}, \alpha<1$, corresponds to an irregular tessellation. The decrease in the regularity parameter $\delta$ corresponds to more disordered VT. Fig 4 shows the random Voronoi cell generated for different regularity parameters $(\alpha)$.

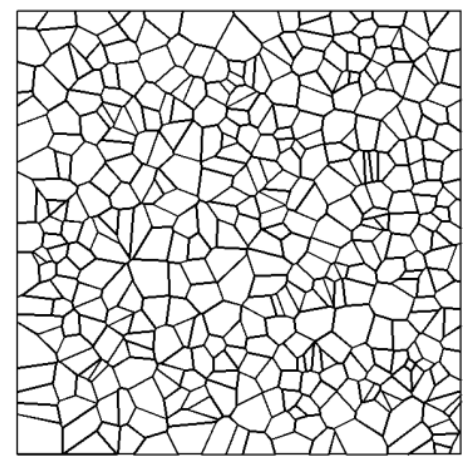

(a)

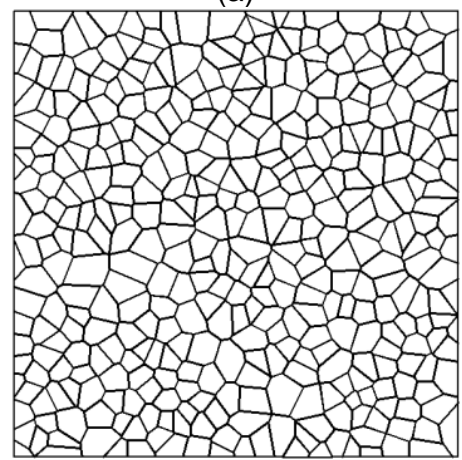

(c)

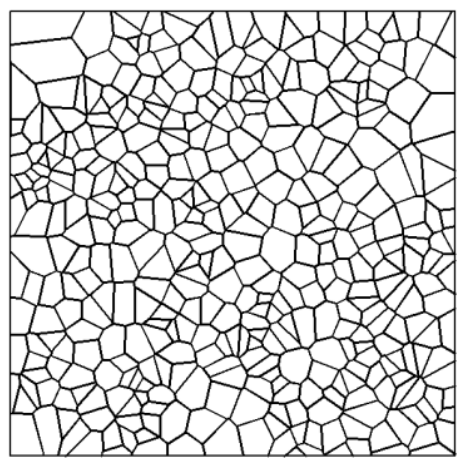

(b)

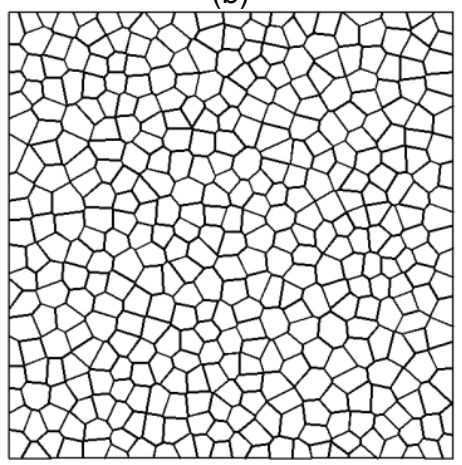

(d)

Fig 4. Randomly generated Voronoi tessellation for different regularity parameters

(a) $\alpha=0$ (b) $\alpha=0.3$ (c) $\alpha=0.5$ (d) $\alpha=0.7$

The area distribution of the Voronoi tessellation for different regularity parameters are shown in Fig 5 . For a Poisson Voronoi tessellation $\alpha=0$, the broader distribution is obtained. On the other hand the shape of the distribution function becomes narrower with the increase in the regularity parameter. The effect of regularity increases with the increase in the $\alpha$ parameter and is shown in Fig 6. A lognormal distribution function has been employed to model grain size distribution features for 2D Voronoi tessellations with different regularities.
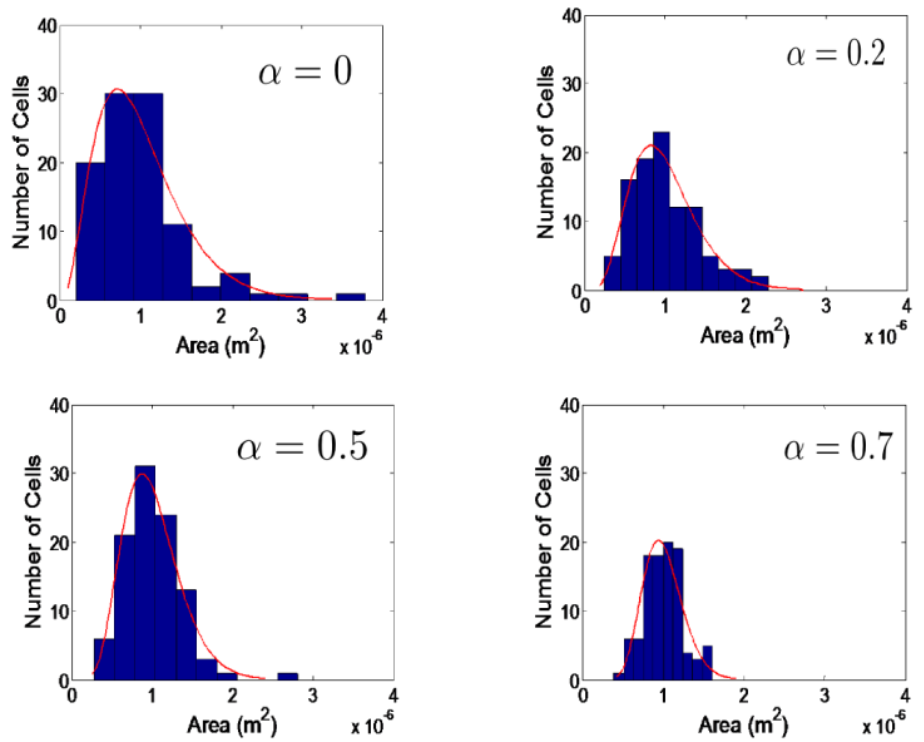

Fig 5 Voronoi cell area distribution for different regularity parameters 


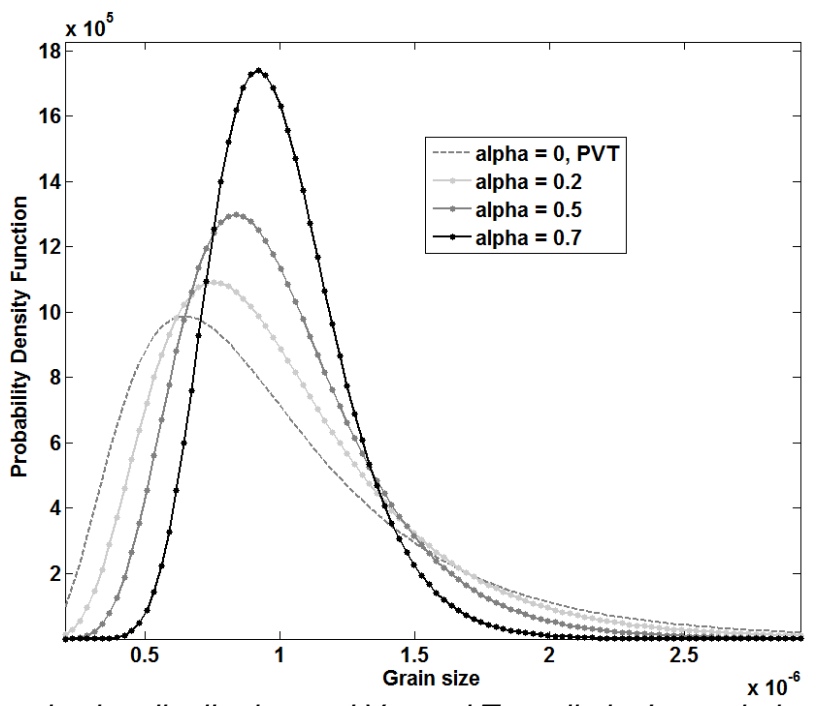

Fig 6 Relation between grain size distribution and Voronoi Tessellation's regularity parameter modeled by a lognormal distribution function

The second important factor that affect the properties of anisotropic material is texture. For a discrete microstructure containing moderate number of grains, the effect of texture is much more important than the grain size effect [13], [2]. In order to examine the property of the microstructure on the different texture, a random field texture is considered, with grain orientation as variable. We assume that every grain has equal probability to be oriented in any direction. In this 2D model, the orientation of individual grain is defined by corresponding rotation around crystal coordinate axis. Fig 7 shows the oriented grains in a Voronoi tessellation. The property tensor calculation based on the global coordinate system is as follows

$$
K_{G}=R K_{L} R^{T}
$$

Where $K_{L}$ is the property tensor in the local coordinate system and $R$ is the rotation matrix as follows

$$
R=\left[\begin{array}{cc}
\cos \theta & \sin \theta \\
-\sin \theta & \cos \theta
\end{array}\right]
$$

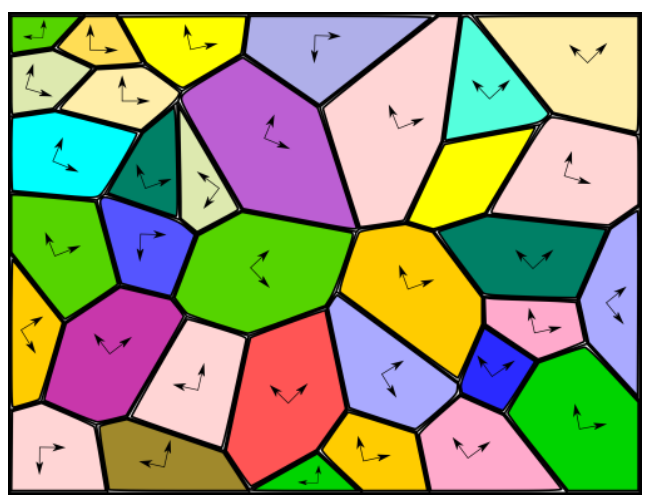

Fig 7 Random orientations represented by local coordinate axis for individual grains in a polycrystalline material

\section{Modeling of Heat diffusion in Polycrystalline Microstructure}

The finite element based heat diffusion phenomena is modeled using the commercial software package COMSOL Multiphysics. The MATLAB - COMSOL live link is used to assign the random thermal conductivity tensor for each grain. The computational domain size is restricted to $50 \mathrm{~mm} \times 50 \mathrm{~mm}$. A point heat source located at the geometric center of the domain. The materials considered for the problem are polycrystalline with different anisotropy ratio, as reported in Ref. [14]. The lateral surface of the unbounded microstructural domain that represent the truncation of an 
infinite extent is studied in two different approach to avoid any reflections occurring from the domain boundary. In the first case a adiabatic boundary condition is used by considering a time duration till the heat reaches the lateral boundary. For the second case the absorbing boundary conditions are imposed using infinite element concept. We consider three different internal boundary conditions for the Voronoi edges: a heat continuity, thermally thin resistive layer (TTRL) and a highly conductive layer (HCL).

\section{Results}

Figure Fig 8 and Fig 9 below presents the computational results for a 10-cell domain obtained after 50 trials (each trial consists of specifying a random set of orientation angles by which the thermal conductivity tensor is transformed and assigned to each cell). The figures on the left hand side indicate the time temperature profiles for two different probes placed equally away $(15 \mathrm{~mm})$ from the heat source in both horizontal and vertical directions. The thick lines represent the outcome of heat diffusion in a single anisotropic cell occupying the entire computational domain. The dotted lines indicate the outcomes of each trial on the 10-cell domain. The black thick lines corresponds to the average values for two different probes.

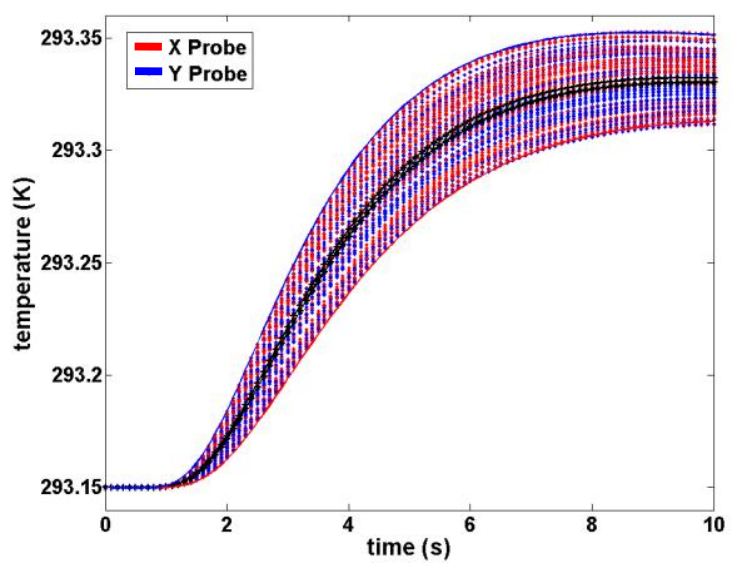

Fig 8 Averaged thermal profiles for a 10-cell domain obtained after 50 trials

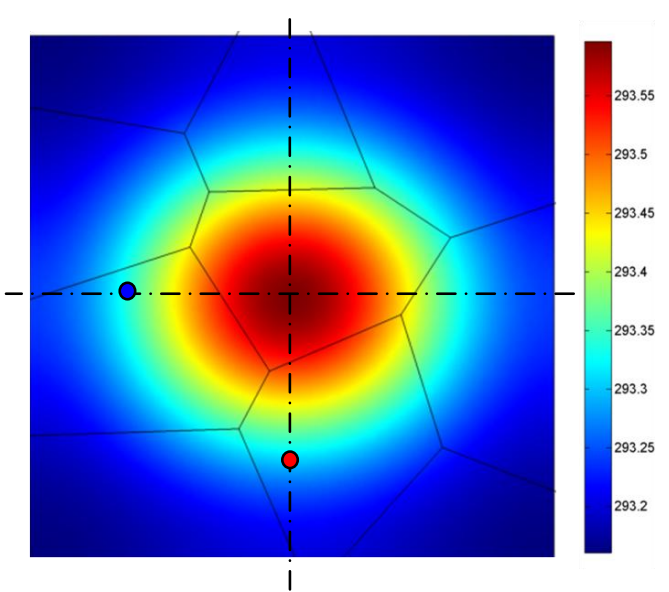

Fig 9 Temperature distribution at the end of computational run for a 10-cell domain

From Fig 9 we can see that the heat is passing trough only one orientation (cell) before reaching the probes. So for each randomly generated orientation gives a more spread in the temperature profiles in the upper and lower bound of the corresponding anisotropic single cell (thick lines) as shown in Fig 8.

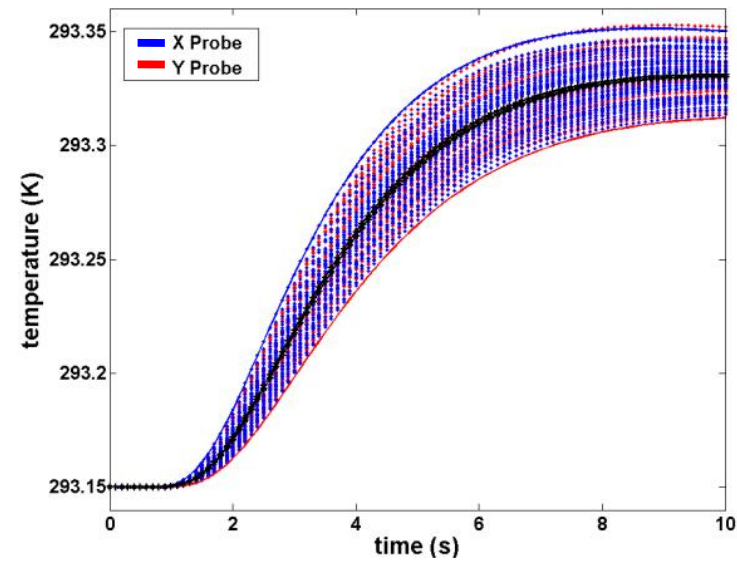

Fig 10 Averaged thermal profiles for a 20-cell domain obtained after 50 trials

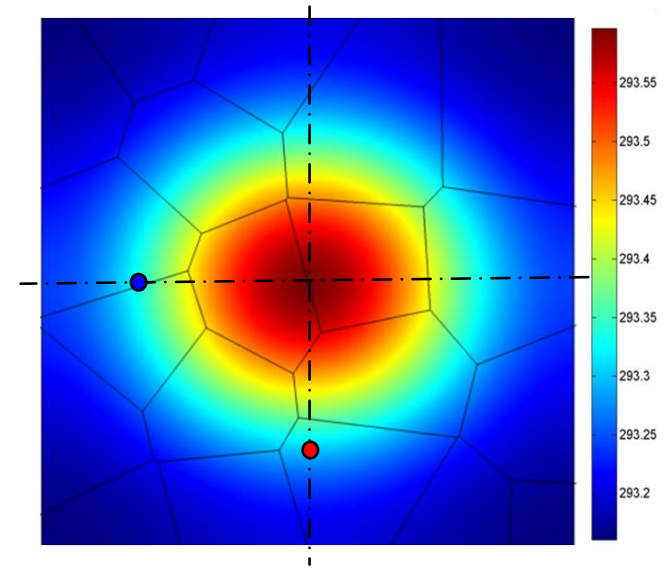

Fig 11 Temperature distribution at the end of computational run for a 20 -cell domain

Fig 10 and Fig 11 presents the computational results for a 20-cell domain obtained after 50 trials. In this case the heat propagation is taking place through two orientations and the spread looks almost similar to that of 10-cell case. 


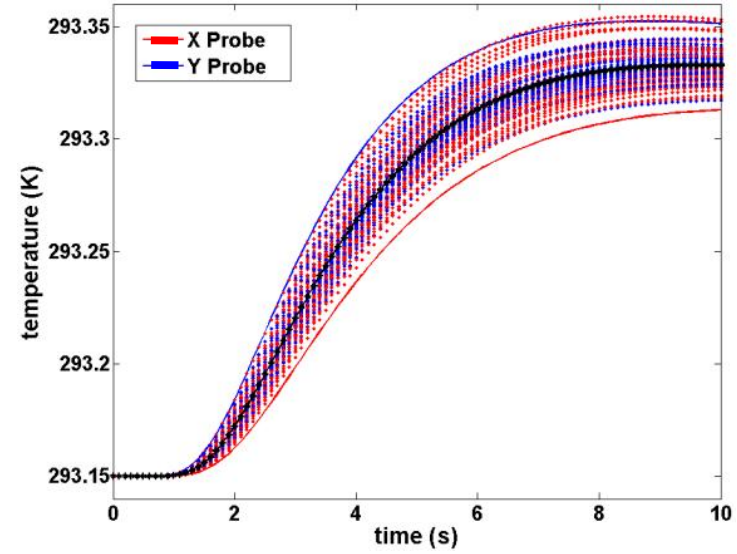

Fig 12 Averaged thermal profiles for a 50-cell domain obtained after 50 trials

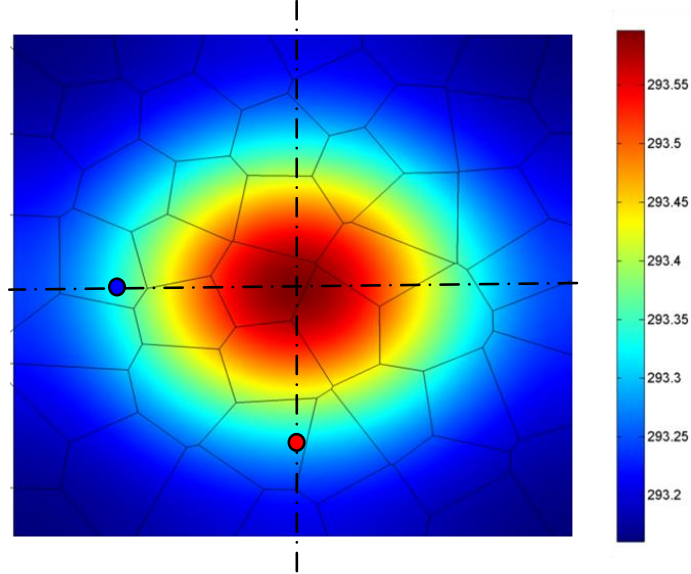

Fig 13 Temperature distribution at the end of computational run for a 50 -cell domain

Fig 12 and Fig 13 presents the computational runs for a 50-cell domain obtained after 50 trials. In this case there observed a slightly more shift towards the average value from the Y probe. Fig 14 and Fig 15 corresponds to the 100-cell domain obtained after 50 trials. As the heat passing through more number of grains, the spread around the average value reduces and can be seen from Fig 12. Fig 16 and Fig 17 shows the computational results for a 150-cell domain and Fig 18 and Fig 19 for the 200-cell domain obtained after 50 trials. It is observed that the spread in the temperature is decreasing with increase in the heat flux interaction with more grain orientations.

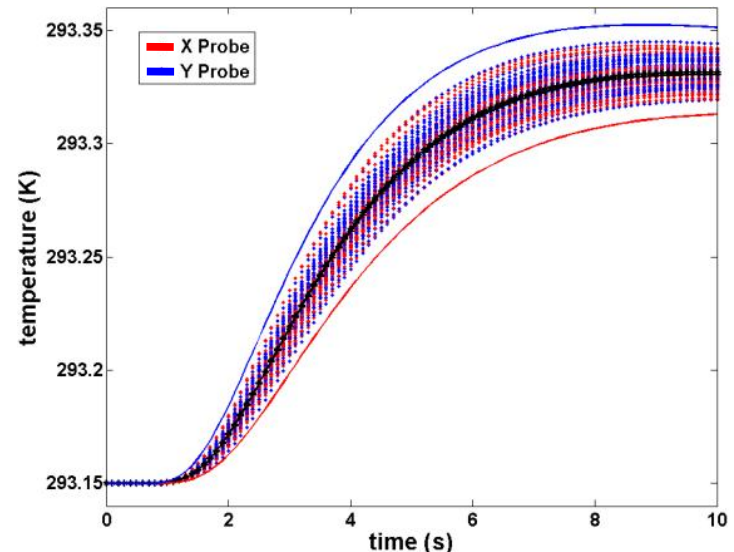

Fig 14 Averaged thermal profiles for a 100-cell domain obtained after 50 trials

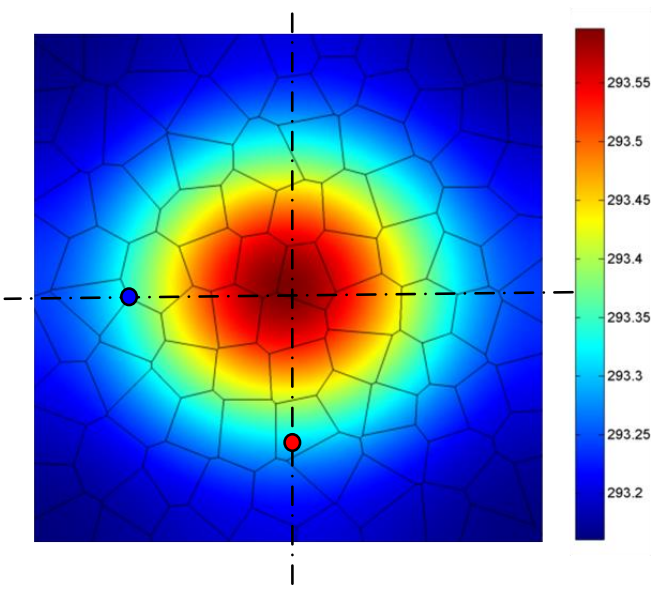

Fig 15 Temperature distribution at the end of computational run for a 100-cell domain 


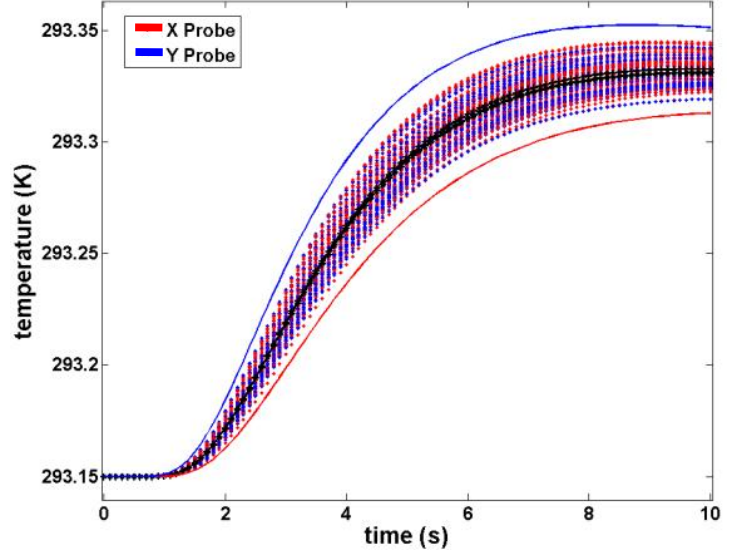

Fig 16 Averaged thermal profiles for a 150-cell domain obtained after 50 trials

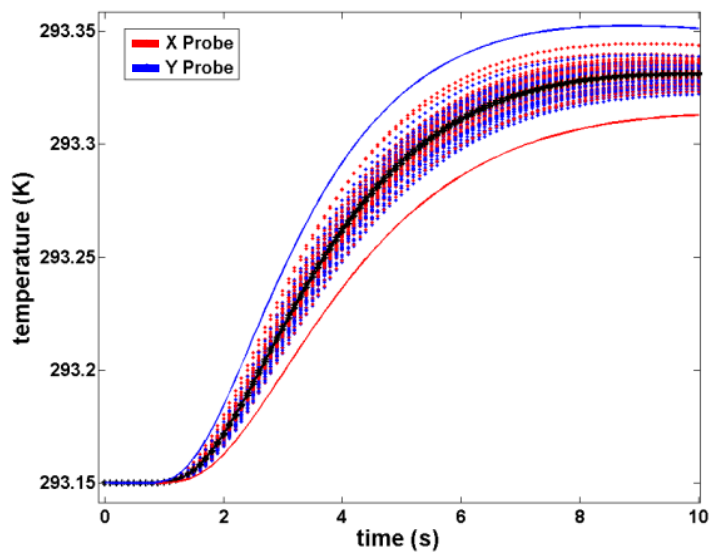

Fig 18 Averaged thermal profiles for a 200-cell domain obtained after 50 trials

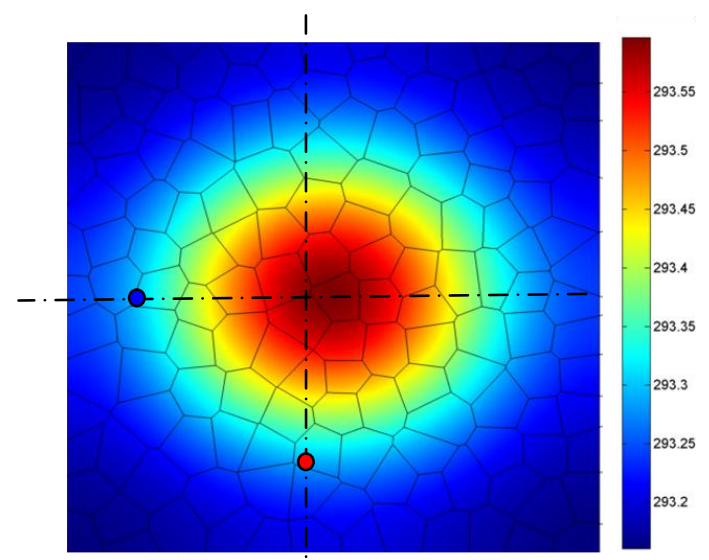

Fig 17 Temperature distribution at the end of computational run for a 150-cell domain

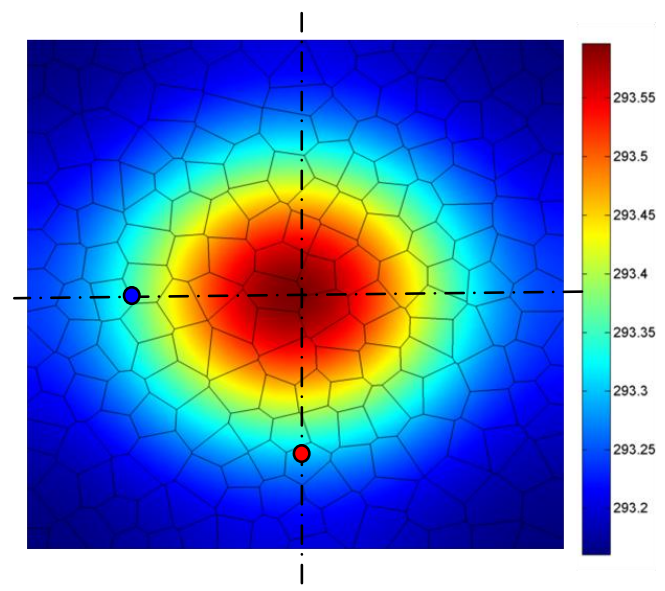

Fig 19 Temperature distribution at the end of computational run for a 200-cell domain

Similarly the two other boundary conditions, Thin Thermally Resistive Layer (TTRL) and Highly conductive layer $(\mathrm{HCL})$ were also used to find the orientational averaged heat diffusion in the polycrystalline material. Figure 20 shows the average temperature plot for a 100-cell domain obtained after 50 trials for the three different boundary conditions. In this case the anisotropy ratio of the material was chosen as 2.39. Further investigation on the different parameters used for the boundary conditions are necessary to get better understanding of the inter cell boundary interactions.

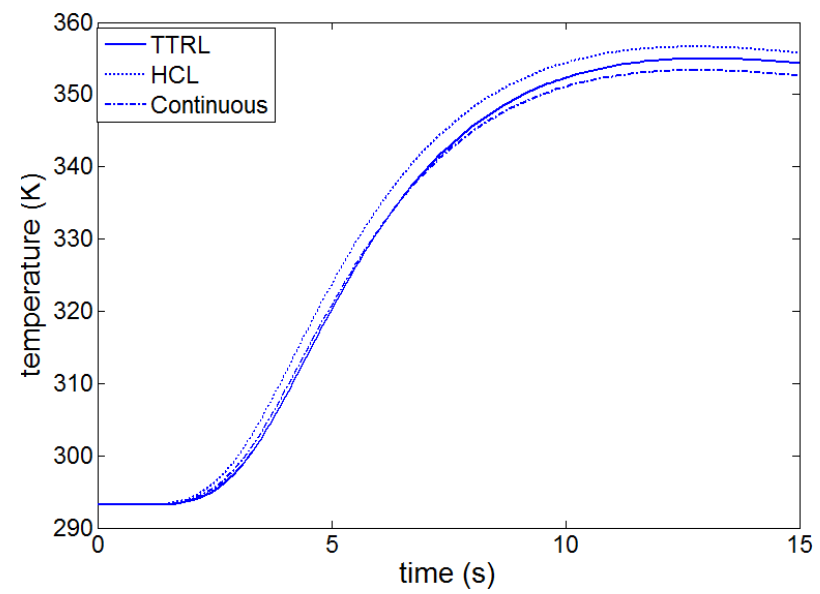

Figure 20 Average temperature plot for a 100-cell domain obtained after 50 trials for three different inter cell boundary conditions 


\section{Summary and Conclusion}

A 2D Voronoi cell tessellation model is proposed for representing randomly oriented anisotropic single crystal grains in polycrystalline material. A regularity parameter for the controlled Voronoi cell generation is studied in this work. Simulation of heat diffusion, based on FEM, is carried out using MATLAB with COMSOL Multiphysics. Heat diffusion is studied for various size distributions and crystallographic orientations of individual cells. Effective anisotropy is monitored over various trials using two orthogonally placed probes with a centrally heated spot heating source. Three types of boundary conditions are considered to deal with heat transport across cells. Results indicate the possibility of representing an anisotropic polycrystalline material by an effective medium under certain conditions.

\section{REFERENCES}

[1] I. Benedetti and M. H. Aliabadi, "A three-dimensional grain boundary formulation for microstructural modeling of polycrystalline materials," Comput. Mater. Sci., vol. 67, pp. 249-260, Feb. 2013.

[2] B. Wen, Z. Li, and N. Zabaras, "Thermal Response Variability of Random Polycrystalline Microstructures," Commun. Comput. Phys., vol. 10, no. 3, pp. 607-634, 2011.

[3] Z. Aksamija and I. Knezevic, "Lattice thermal transport in large-area polycrystalline graphene," Phys. Rev. B, vol. 90, no. 3, p. 035419, Jul. 2014.

[4] I. Srivastava, S. Sadasivam, K. C. Smith, and T. S. Fisher, "Combined Microstructure and Heat Conduction Modeling of Heterogeneous Interfaces and Materials," J. Heat Transfer, vol. 135, no. 6, p. 061603, May 2013.

[5] J. Amrit, "Grain boundary Kapitza resistance and grain-arrangement induced anisotropy in the thermal conductivity of polycrystalline niobium at low temperatures," J. Phys. D. Appl. Phys., vol. 39, no. 20, pp. 44724477, Oct. 2006.

[6] P. Alveen, D. Carolan, D. McNamara, N. Murphy, and a. Ivanković, "Micromechanical modelling of ceramic based composites with statistically representative synthetic microstructures," Comput. Mater. Sci., vol. 79, pp. 960-970, Nov. 2013.

[7] Z. Fan, Y. Wu, X. Zhao, and Y. Lu, "Simulation of polycrystalline structure with Voronoi diagram in Laguerre geometry based on random closed packing of spheres," Comput. Mater. Sci., vol. 29, no. 3, pp. 301-308, Mar. 2004.

[8] L. Lin, X. Wang, and X. Zeng, "Geometrical Modeling of Cell Division and Cell Remodeling Based on Voronoi Tessellation Method," vol. 98, no. 2, pp. 203-220, 2014.

[9] H. X. Zhu, P. Zhang, D. Balint, S. M. Thorpe, J. a. Elliott, a. H. Windle, and J. Lin, "The effects of regularity on the geometrical properties of Voronoi tessellations," Phys. A Stat. Mech. its Appl., vol. 406, pp. 42-58, Jul. 2014.

[10] J. Cho, "Voronoi diagram and microstructure of weldment," J. Mech. Sci. Technol., vol. 29, no. 1, pp. 371-374, Jan. 2015.

[11] P. Zhang, M. Karimpour, D. Balint, J. Lin, and D. Farrugia, "A controlled Poisson Voronoi tessellation for grain and cohesive boundary generation applied to crystal plasticity analysis," Comput. Mater. Sci., vol. 64, pp. 84-89, Nov. 2012.

[12] H. X. Zhu, S. M. Thorpe, and a. H. Windle, "The geometrical properties of irregular two-dimensional Voronoi tessellations," Philos. Mag. A, vol. 81, no. 12, pp. 2765-2783, Sep. 2007.

[13] F. Yang, T. Ikeda, G. J. Snyder, and C. Dames, "Effective thermal conductivity of polycrystalline materials with randomly oriented superlattice grains," J. Appl. Phys., vol. 108, no. 3, p. 034310, 2010.

[14] a. Tiwari, K. Boussois, B. Nait-Ali, D. S. Smith, and P. Blanchart, "Anisotropic thermal conductivity of thin polycrystalline oxide samples," AIP Adv., vol. 3, no. 11, p. 112129, 2013. 
http://dx.doi.org/10.21611/qirt.2015.0072 